dose-dependently trigger cell proliferation. The stability of a lentiviral vector-induced, truncated HMGA2 mRNA that was deleteriously increased by loss of miRNA target sequences is a sobering counterexample (3), yet it suggests approaches for the development of safeguards. For instance, the inclusion of cell type- and stage-specific miRNA target sequences in vectorderived transcripts can elegantly restrict transgene expression to particular targets (16). Properly tailored, it could similarly serve to destabilize harmful fusion transcripts in cells particularly susceptible to transformation, namely stem cells and early precursors. In situations in which only differentiated cells require phenotypic correction for disease to be prevented, the safety margins of integrating gene therapy vectors could thus be significantly increased by combining stage- and lineage-specific promoters, to avoid protooncogene activation in stem cells and early precursors (17), and sequences targeted by miRNAs expressed in these cells, in which they would promote the degradation of dangerous cellular-viral fusion transcripts (Figure 1C and ref. 18). Pending the advent of efficient techniques for site-specific integration and clonal stem cell expansion (19), such tricks may significantly improve the safety of tools currently available for gene- and cell-based therapies.

\section{Acknowledgments}

Work in my laboratory is supported by the Swiss National Science Foundation, the European community (PERSIST), and the European Research Council.

Address correspondence to: Didier Trono, Ecole Polytechnique Fédérale de Lausanne, SV-DO - Station 19, CH-1015, Lausanne, Switzerland. Phone: 41.21.693.1634; Fax: 41. 21.693.1635; E-mail: didier.trono@epfl.ch.

1. Hacein-Bey-Abina S, et al. Efficacy of gene therapy for X-linked severe combined immunodeficiency. N Engl J Med. 2010;363(4):355-364.

2. Cavazzana-Calvo M, et al. Gene therapy of human severe combined immunodeficiency (SCID)-X1 disease. Science. 2000;288(5466):669-672.

3. Cavazzana-Calvo $M$, et al. Transfusion independence and HMGA2 activation after gene therapy of human beta-thalassaemia. Nature. 2010;467(7313):318-322

4. Aiuti A, et al. Gene therapy for immunodeficiency due to adenosine deaminase deficiency. $N$ Engl J Med. 2009;360(5):447-458.

5. Cartier N, et al. Hematopoietic stem cell gene therapy with a lentiviral vector in X-linked adrenoleukodystrophy. Science. 2009;326(5954):818-823.

6. Boztug K, et al. Stem-cell gene therapy for the Wiskott-Aldrich syndrome. $N$ Engl J Med. 2010;363(20):1918-1927.

7. Hacein-Bey-Abina S, et al. A serious adverse event after successful gene therapy for X-linked severe combined immunodeficiency. $N$ Engl J Med. 2003;348(3):255-256.

8. Stein S, et al. Genomic instability and myelodysplasia with monosomy 7 consequent to EVI1 activation after gene therapy for chronic granulomatous disease. Nat Med. 2010;16(2):198-204.
9. Krause D. Gene therapy for Wiskott-Aldrich syndrome: Benefits and risks. The Hematologist. 2011;8(2):10. http://www.hematology.org/Publications/Hematologist/2011/6487.aspx

10. Hacein-Bey-Abina S, et al. LMO2-associated clonal $\mathrm{T}$ cell proliferation in two patients after gene therapy for SCID-X1. Science. 2003;302(5644):415-419.

11. Montini E, et al. The genotoxic potential of retroviral vectors is strongly modulated by vector design and integration site selection in a mouse model of HSC gene therapy. J Clin Invest. 2009;119(4):964-975.

12. Wu X, Li Y, Crise B, Burgess SM. Transcription start regions in the human genome are favored targets for MLV integration. Science. 2003;300(5626):1749-1751.

13. Moiani A, et al. Lentiviral vector integration in the human genome induces alternative splicing and generates aberrant transcripts. J Clin Invest. 2012;122(5):1653-1666

14. Cesana D, Sgualdino J, Rudilosso L, Merella S, Naldini L, Montini E. Whole transcriptome characterization of aberrant splicing events induced by lentiviral vector integrations. $J$ Clin Invest. 2012;122(5):1667-1676.

15. Almarza D, Bussadori G, Navarro M, Mavilio F, Larcher F, Murillas R. Risk assessment in skin gene therapy: viral-cellular fusion transcripts generated by proviral transcriptional read-through in keratinocytes transduced with self-inactivating lentiviral vectors. Gene Ther. 2011;18(7):674-681.

16. Brown BD, Venneri MA, Zingale A, Sergi Sergi L, Naldini L. Endogenous microRNA regulation suppresses transgene expression in hematopoietic lineages and enables stable gene transfer. Nat Med. 2006;12(5):585-591.

17. Barde I, et al. Lineage- and stage-restricted lentiviral vectors for the gene therapy of chronic granulomatous disease. Gene Ther. 2011;18(11):1087-1097.

18. Brown BD, et al. Endogenous microRNA can be broadly exploited to regulate transgene expression according to tissue, lineage and differentiation state. Nat Biotechnol. 2007;25(12):1457-1467.

19. Naldini L. Ex vivo gene transfer and correction for cellbased therapies. Nat Rev Genet. 2011;12(5):301-315.

\title{
Epidermal Langerhans cells tune skin reactivity to contact allergens
}

\author{
Mark C. Udey
}

Dermatology Branch, Center for Cancer Research, National Cancer Institute, NIH, Bethesda, Maryland, USA.

\begin{abstract}
Allergic contact dermatitis is a common disorder that has fascinated dermatologists and immunologists for decades. Extensive studies of contact sensitivity reactions in mice established a mechanistic paradigm that has been revisited in recent years, and the involvement of Langerhans cells (LCs), a population of epidermal dendritic cells, in immune responses to epicutaneously applied antigens has been questioned. In this issue of the JCI, Gomez de Agüero et al. describe an elegant series of experiments that implicate LCs in tolerance induction, positioning these cells as key regulators of immunologic barrier function.
\end{abstract}

Conflict of interest: The author has declared that no conflict of interest exists.

Citation for this article: $J$ Clin Invest. 2012;122(5):1602-1605. doi:10.1172/JCI63190.
Skin represents a dynamic, responsive interface that separates organism and environment (reviewed in ref. 1). Epidermal keratinocytes that are capped by a nonvital cornified layer and joined by a network of tight junctions constitute a physical barrier that, under normal circumstances, prevents entry of many environmental agents. An increasingly well-defined constellation of immune and inflammatory cells creates an immunologic barrier that is poised to respond to environmental insults that breach the skin's physical barrier. Reactivity of this immunologic barrier is fine tuned. Pathogenic microbes trigger responses that are sufficiently vigorous and sustained so that offending organisms are contained and ultimately cleared without 
A All individuals

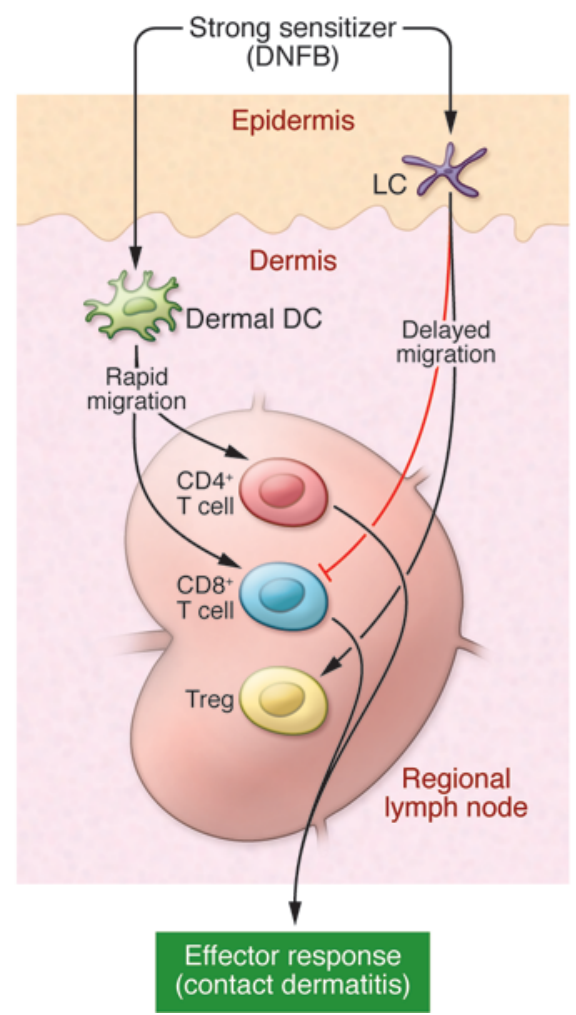

B Nonallergic individuals

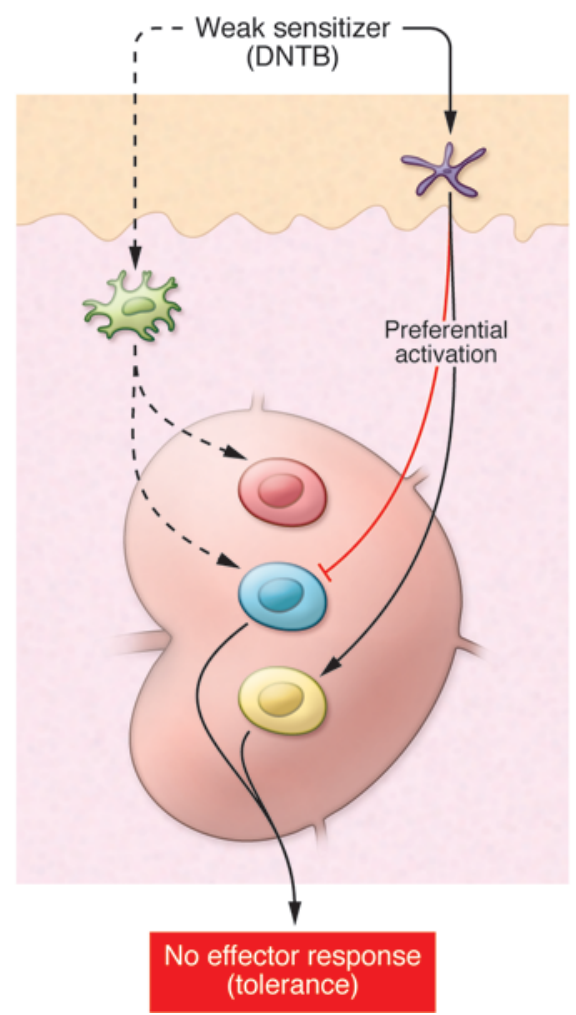

C Allergic individuals

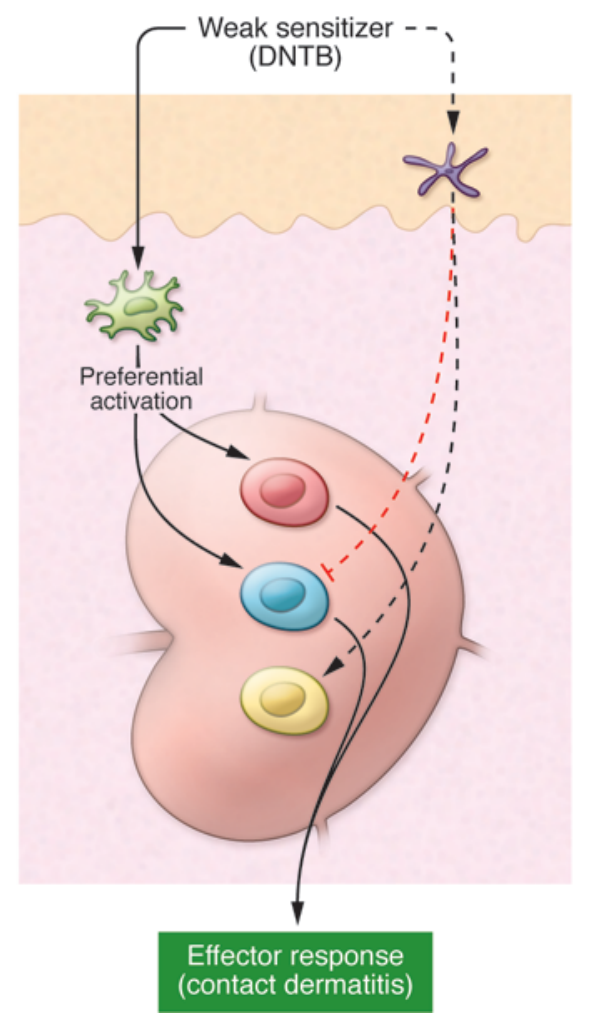

Figure 1

A suggested role for epidermal LCs in contact dermatitis susceptibility. (A) Strong sensitizers activate LCs and dermal DCs (dDC), but dDCs preferentially stimulate effector immune responses that cause contact dermatitis. (B) In nonallergic individuals, weak sensitizers preferentially activate LCs that stimulate Tregs and anergize CD8 ${ }^{+}$T cells, leading to tolerance of the weak sensitizer. (C) In allergic individuals, LC dysfunction may allow even suboptimally activated dDCs to initiate contact sensitivity reactions (contact dermatitis).

unwanted collateral damage. Innocuous environmental constituents that penetrate the skin's physical barrier may be tolerated either because they are ignored by the immunologic barrier or because they trigger counterbalancing immunoregulatory mechanisms that prevent inflammation.

Allergic contact dermatitis is a common skin disease that can be related to perturbations of skin's physical and immunologic barriers (reviewed in ref. 2). Contact dermatitis (both irritant and allergic forms) is, by far, the most common occupationrelated disorder, and the direct and indirect costs to society are considerable (2). Common causes of allergic contact dermatitis in humans include metals, preservatives, fragrances, and chemicals involved in the manufacture of rubber: substances that are ubiquitous in the environment in the developed world. Most causes of allergic contact dermatitis in patients are said to be "weak sensitizers" because they do not cause dermatitis in all exposed individuals and, even in allergic individuals, sensitization may require many exposures. Understanding how healthy individuals tolerate weak sensitizers will provide important insights into the pathophysiology of contact dermatitis.

\section{Modeling allergic contact dermatitis}

Because it is not amenable to study in vitro, for many years, allergic contact dermatitis has been modeled in small animals (reviewed in ref. 3). In a typical experiment, mice are sensitized by application of a chemically reactive small molecule (e.g., dinitrofluorobenzene [DNFB]) to abdominal skin, and inflammatory responses are subsequently elicited by applying the sensitizing chemical onto ear skin a few days later. Chemicals that are used in these studies are typically strong sensitizers that induce responses in all mice of a given strain and in most mouse strains. These sensitizers are small (<500 kDa), lipophilic, and chemically reactive, and thus they readily distribute beyond application sites and react with proteins and peptides that may be on (or in) many different cells forming a variety of adducts. For these reasons, the precise antigens that are recognized during the sensitization and elicitation phases of contact sensitivity reactions are not known and identification of the cells and cell products that are critical regulators of contact sensitivity has been challenging.

Experiments conducted by multiple groups established a mechanistic paradigm of contact sensitivity reactions (3) wherein application of a contact sensitizer induces antigen loading and activation of cutaneous DCs, followed by migration of these APCs to regional lymph nodes where they encounter naive $\mathrm{CD}^{+}$and $\mathrm{CD}^{+} \mathrm{T}$ cells. In lymph nodes, $\mathrm{CD}^{+}$and $\mathrm{CD}^{+} \mathrm{T}$ cells proliferate and differentiate, subsequently entering the peripheral blood. Circulating functionally competent effector $\mathrm{T}$ cells are able to home to sites of antigen exposure where they can initiate immune reactions manifested as contact dermatitis in humans or ear swelling and inflammation in mice. 


\section{Changing the mechanistic paradigm}

This construct is simplistic on multiple levels and ignores or underemphasizes the involvement and importance of many cells (including keratinocytes, mast cells, NK cells, and B cells) and an array of proinflammatory and/or immunoregulatory cytokines and chemokines and associated downstream signaling molecules as well as other soluble mediators, including complement proteins and other proteases. Recently recognized phenotypic heterogeneity of skin DCs was also not taken into account.

Epidermal Langerhans cells (LCs) represent the most extensively studied skin DC subpopulation, and all DCs in unperturbed epidermis are LCs. In contrast, there are multiple DC subpopulations in mammalian dermis, and these cells are distinct from LCs (4-6). Experimentally, LCs can be differentiated from dermal DCs based on their expression of the C-type lectin Langerin and several surface markers, relative radioresistance, slow turnover, and TGF- $\beta$ dependence. Complicating these lineage definitions, a small subpopulation of dermal DCs in mice that are not related to LCs expresses Langerin (4-6).

In the contact hypersensitivity paradigm described above, it was presumed that, because of their location in the outermost layer of skin (epidermis) and their potency as APCs in vitro and after adoptive transfer in vivo, LCs actively participated in generation of effector immune responses. Development of transgenic mice that were LC deficient by three separate groups (7-9) allowed formal testing of this hypothesis. Surprisingly, none of the initial experiments indicated that LCs were absolutely required during sensitization or elicitation phases of contact sensitivity reactions. Two of the models involved depletion of Langerin-expressing DCs and LCs $(7,8)$, and the modest decrements in inflammation observed can now be attributed to involvement of dermal DCs in T cell priming to contact allergens (reviewed in ref. 10). In the other model (in which only LCs were absent), contact sensitivity to DNFB was actually increased approximately 2 -fold (9). In a follow-up study, attenuation of DNFB-induced responses by LCs required that LCs express MHC class II antigens and produce IL-10, the former implying a requisite physical interaction between LCs and CD4 ${ }^{+} \mathrm{T}$ cells $(11)$

Judicious study of mice deficient in LCs and Langerin-expressing dermal DCs has subsequently resulted in a number of important insights into cutaneous DC function. The essential involvement of LCs in contact sensitivity reactions (10), skin graft rejection (12), reactivity to skin self antigens (13), graft-versus-host disease (14), several skin infections (including herpes simplex, ref. 15; leishmaniasis, ref. 16; and candidiasis, ref. 17) and antibody production in response to cutaneous antigen exposure $(18,19)$ has been excluded, and instead, an immunomodulatory role for LCs has emerged. In addition to the initial report that documented enhanced contact sensitivity responses in mice lacking only LCs (10), subsequent studies have implicated LCs in tolerance to minor histocompatibility antigens (12), generation of Th17 cells (17), attenuation of antiparasite responses (16), and regulation of isotype utilization in antibody responses $(18,19)$. In this issue of the JCI, Gomez de Agüero and coworkers extend these observations, convincingly implicating LCs in tolerance to a contact allergen (20).

\section{LCs can promote tolerance}

It has been long known that although DNFB and 2,4-dinitrothiocyanobenzene (DNTB) are cross-reactive with respect to murine T cells, DNFB is a strong sensitizer, while immunization with DNTB required multiple exposures to high concentrations of reagent (21). Interestingly, one epicutaneous exposure of mice to DNTB renders animals tolerant to subsequent immunization with DNFB (22), even if the DNTB and DNFB are applied to different sites. Gomez de Agüero et al. demonstrate that, somewhat surprisingly, DNTB efficiently mobilized LCs from skin to lymph nodes in the absence of skin irritation (20). Although other skin DCs (especially Langerin ${ }^{-}$dermal DCs) were also mobilized by DTNB, in lymph nodes, LCs carried the highest density of dinitrophenyl epitopes. Depletion of LCs from skin prior to DNTB treatment abrogated tolerance induction, indicating that LCs were essential for this process.

That LCs were sufficient for tolerance induction was demonstrated in experiments in which a remarkably small number $(\sim 25,000)$ of DTNB-treated LCs tolerized naive recipients after in vivo administration. This activity required MHC class I antigen expression by LCs, suggesting that direct contact between LCs and $\mathrm{CD}^{+}$ $\mathrm{T}$ cells might be required $-\mathrm{a}$ conclusion that was bolstered by the observation that naive $\mathrm{CD}^{+} \mathrm{T}$ cells could be anergized by incubation with DNTB-modified LCs in vitro. Regulatory $\mathrm{T}$ cells apparently also play a role in DTNB-induced tolerance, since DNTB application or administration of DNTB-treated LCs led to Treg activation and DNTB-induced tolerance did not occur in Treg-deficient mice (20).

\section{Therapeutic implications}

This work demonstrates that presentation of antigen to $\mathrm{T}$ cells by LCs can dampen reactivity to subsequent exposure to a potent structurally related immunogen. Whether or not these observations will apply to other chemicals and to humans remains to be determined, but regardless, it is attractive to speculate that small molecules with selected characteristics of DNTB (e.g., ability to mobilize LCs without activating inflammasomes [ref. 23] and inducing proinflammatory cytokine production) might have unique antiinflammatory properties and clinical utility in patients with contact dermatitis or other eczematous disorders. Development of these types of candidate therapeutics will require a more detailed understanding of how migration of the various skin DC subsets is regulated.

An additional question that is raised by these experiments is whether or not the biologic activity of LCs can be differentially regulated. Is DNTB tolerogenic because epicutaneous application induces an LC activation state that is different from that induced by DNFB, or do these two structurally related molecules differ with respect to immunogenicity because they mobilize different DC subpopulations to different extents or with different kinetics? Do these molecules differentially induce one or more proinflammatory cytokines? Additional studies will be required to address these issues.

Finally, it is interesting to note that, even in this simple experimental system, there is redundancy in the immunoregulatory mechanisms that are invoked: DNTB acts via LCs to downmodulate inflammation by anergizing $\mathrm{CD}^{+} \mathrm{T}$ cells and also by activating Tregs. The existence of redundancy in biologic systems often highlights the importance of the process under consideration. One can conceptualize the skin immunologic barrier as a tunable biologic system, poised to react to insults from the outside world without overreacting. This biologic system is able to distinguish self from non-self, to regulate the intensity and duration of protective inflammatory reactions, and to limit the development and severity of injurious reactions to innocuous environmental constituents. The results of 
Gomez de Agüero and coworkers suggest that LCs may help tune the reactivity of the skin's immunologic barrier and that LC dysfunction may contribute to development of allergic contact dermatitis to ubiquitous weak sensitizers (Figure 1).

\section{Acknowledgments}

I thank Stephen I. Katz for helpful discussions. I am supported by the Intramural Research Program (IRP) of the Center for Cancer Research, National Cancer Institute, NIH.

Address correspondence to: Mark C. Udey, Dermatology Branch, CCR, NCI, NIH, Building 10, Room 12N238, Bethesda, Maryland 20892-1908, USA. Phone: 301.496.2481; Fax: 301.496.5370; E-mail: udey@helix.nih.gov.

1. Kubo A, Nagao K, Amagai M. Epidermal barrier dysfunction and cutaneous sensitization in atopic disease. J Clin Invest. 2012;122(2):440-447.

2. Cashman MW, Reutemann PA, Ehrlich A. Contact dermatitis in the United States: Epidemiology, economic impact, and workplace prevention. Dermatol Clin. 2012;30(1):87-98.

3. Kaplan DH, Igyarto BZ, Gaspari AA. Early immune events in the induction of allergic contact dermati- tis. Nature Rev Immunol. 2012;12(2):114-124.

4. Merad M, Ginhoux F, Collin M. Origin, homeostasis and function of Langerhans cells and other langerin-expressing dendritic cells. Nature Rev Immunol. 2008;8(12):935-947

5. Guilliams M, et al. DC lineage and subsets. Eur J Immunol. 2010;40(8):2085-2130.

6. Henri S, et al. Disentangling the complexity of the skin dendritic cell network. Immunol Cell Biol. 2010;88(4):366-375.

7. Bennett CL, et al. Inducible ablation of mouse Langerhans cells diminishes but fails to abrogate contact hypersensitivity. J Cell Biol. 2005;169(4):569-576.

8. Kissenpfennig A, et al. Dynamics and function of Langerhans cells in vivo: Dermal dendritic cells colonize lymph node areas distinct from slower migrating Langerhans cells. Immunity. 2005;22(5):643-654.

9. Kaplan DH, Jenison MC, Saeland S, Shlomchik WD, Shlomchik MJ. Epidermal Langerhans celldeficient mice develop enhanced contact hypersensitivity. Immunity. 2005;23(6):611-620.

10. Kaplan DH, Kissenpfennig A, Clausen BE Insights into Langerhans cell function from Langerhans cell ablation models. Eur J Immunol. 2008; 38(9):2369-2376

11. Igyarto BZ, et al. Langerhans cells suppress contact hypersensitivity responses via cognate CD4 interaction and Langerhans cell-derived IL-10. J Immunol. 2009;183(8):5085-5093.

12. Obhrai JS, et al. Langerhans cells are not required for efficient skin graft rejection. J Invest Dermatol. 2008;128(8):1950-1955

13. Bursch LS, Rich BE, Hogquist KA. Langerhans cells are not required for the CD8 T cell response to epidermal self-antigens. JImmunol. 2009;182(8):4657-4664.
14. Li H, et al. Langerhans cells are not required for graftversus-host disease. Blood. 2011;117(2):697-707.

15. Allan RS, et al. Epidermal viral immunity induced by CD $8 \alpha+$ dendritic cells but not by Langerhans cells. Science. 2003;301(5641):1925-1928.

16. Kautz-Neu K, et al. Langerhans cells are negative regulators of the anti-Leischmania response. J Exp Med. 2011;208(5):885-8891.

17. Igyarto BZ, et al. Skin-resident murine dendritic cell subsets promote distinct and opposing antigen-specific $\mathrm{T}$ helper cell responses. Immunity. 2011:35(2):260-272.

18. Nagao K, et al. Murine epidermal Langerhans cells and langerin-expressing dermal dendritic cells are unrelated and exhibit distinct functions. Proc Natl Acad Sci U S A. 2009;106(9):3312-3317.

19. Ouchi $\mathrm{T}$, et al. Langerhans cell antigen capture through tight junctions confers preemptive immunity in experimental staphylococcal scalded skin syndrome. J Exp Med. 2011;208(13):2607-2613.

20. Gomez de Agüero M, et al. Langerhans cells protect from allergic contact dermatitis in mice by tolerizing $\mathrm{CD}^{+} \mathrm{T}$ cells and activating Foxp $3^{+}$regulatory T cells. J Clin Invest. 2012;122(5):1700-1711.

21. Dearman RJ, Cumberbatch M, Hilton J, Fielding $\mathrm{H}$, Basketter KI. A re-appraisal of skin-sensitizing activity of 2, 4-dinitrothiocyanobenzene. Food Chem Toxicol. 1997;35(2):261-269.

22. Iijima M, Katz SI. Specific immunologic tolerance to dinitroflurobenzene following topical application of dinitrothicyanobezene: Modulation by suppressor T cells. J Invest Dermatol. 1983;81(4):325-330.

23. Watanabe $\mathrm{H}$, et al. Danger signaling through the inflammasome acts as a master switch between tolerance and sensitization. J Immunol. 2008; 180(9):5826-5832.

\title{
Treating myeloma cast nephropathy without treating myeloma
}

\author{
Nelson Leung \\ Division of Nephrology and Hypertension, Mayo Clinic, Rochester, Minnesota, USA.
}

\begin{abstract}
Cast nephropathy is the result of coprecipitation of immunoglobulin free light chains (FLCs) with Tamm-Horsfall glycoprotein (THP). It is a hallmark of multiple myeloma that has significant consequences. Treatment strategies in the past focused on reduction of serum FLC by control of the myeloma. In this issue, Ying et al. report on their successful synthesis of a cyclized competitor peptide that blocks the binding of FLC to THP. In animal studies, this cyclized peptide was capable of reducing cast formation and kidney injury, representing a novel treatment strategy for cast nephropathy that does not depend on the responsiveness of the myeloma to chemotherapy.
\end{abstract}

Sixty-one years passed between the description of Bence Jones proteinuria and the first use of the term "myeloma kidney," by Alfred von Decastello in 1909, to describe the tubu-

Conflict of interest: The author has declared that no conflict of interest exists.

Citation for this article: $J$ Clin Invest.

2012;122(5):1605-1608. doi:10.1172/JCI63248. lar plugging by an amorphous substance in the kidney of myeloma patients (1). Oliver's "cast nephropathy" later replaced it, in 1945, and remains in use today (2). So why was there such a delay in recognizing the nephrotoxic potential of Bence Jones protein $(\mathrm{BJP})$ ? In modern times, renal impairment is accepted as a frequent occurrence in myeloma patients and is one of the diagnostic criteria of symptomatic multiple myeloma (3). The reason that physicians in the late 19th century failed to recognize the nephrotoxicity of BJP can at least be partly attributed to Thomas McBean, the now-famous patient whose urine was studied by William Macintyre, Thomas Watson, and Henry Bence Jones, who had grossly and microscopically normal kidneys despite excreting a calculated $67 \mathrm{~g} / \mathrm{d}$ of protein (4). The same phenomenon has been reported in modern literature, confirming that at least in some patients, a massive amount of Bence Jones proteinuria can have little negative effect on the kidney (5). Part of the mystery was solved in the 1950s by Korngold and Lipari when they discovered BJP actually referred to two different myeloma proteins ( $\kappa$ and $\lambda$, later named in honor of them) (1). Edelman and Gally later identified these proteins as 